\section{Third generation oral contraceptives}

\section{CSM's advice will harm women's health worldwide}

EdrToR,-The Committee on Safety of Medicines' recent advice that oral contraceptives containing gestodene or desogestrel should not be prescribed' has caused confusion and concern among family planning specialists and has major public health implications.

In 1977 the Royal College of General Practitioners concluded that women aged over 35 and heavy smokers aged over 30 should not use oral contraceptives because of an increased risk of cardiovascular disease. ${ }^{2}$ These conclusions were highlighted in the Dutch media and caused a drop in the use of oral contraceptives from $34 \%$ to $27 \%$, resulting in a sudden, $22 \%$ increase in induced abortions followed seven months later by a $6 \%$ increase in the birth rate. I calculated that this sensational media coverage caused some 13000 extra unplanned pregnancies $(40 \%$ ending in induced abortion) in the following year in the Netherlands. It took five years for the use of oral contraceptives and for the abortion rate to return to the rates that existed before the scare. Ironically, induced abortions increased more among younger women than older women (34\% among those aged 20-24 $v 9 \%$ among those aged 35-49). Hence those at increased risk were least affected, while those not at increased risk were disproportionately affected. ${ }^{3}$

Family planning specialists have learnt from such experiences, which is why the Committee on Safety of Medicines' advice causes such concern. It is based only on epidemiological surveys and does not take into account the negative public health impact of such advice. At best, the advice might prevent 1.5 cases of thromboembolism per 10000 woman years, of which less than $1 \%$ are lethal (the thromboembolic risk of pregnancy is double this). Women often pick up the message that "there is something wrong with the pill" and that it is better to stop or not to start using it, with subsequent increases in the abortion and birth rates. Therefore some unofficial "rules" (which the committee ignored) should be adopted by anyone dealing with the outcome of epidemiological studies:

- Do not publicly highlight results until they have been confirmed in several studies

- Do not publicly highlight or draw practical conclusions from studies that focus only on a single risk or benefit. New information should be presented publicly only within the framework of both risks and benefits.

The British advice will undoubtedly influence public opinion and health policies abroad, particularly in developing countries. Recent estimates from the World Health Organisation indicate that 60000-100000 women die annually from the consequences of (illegal) abortion. ${ }^{4}$ This can be prevented by increasing the use of modern contraception. Ill judged messages like that issued by the Committee on Safety of Medicines impede the transition to modern contraception and increase reliance on abortion, causing unnecessary harm to women's health worldwide.

\section{EVERT KETTING} Deputy directo

Netherlands Institute of Social Sexological Research,

Utrecht,

Netherlands

1 Committee on Safety of Medicines. Combined oral contraceptives and thromboembolism. London: CSM, 1995.

2 Royal College of General Practitioners' Oral Contraception Study. Mortality among oral-contraceptive users. Lancet 1977;ii:727-31.

3 Ketting E. De teloorgang van "de pil." Zeist: Netherlands Institute of Social Sexological Research, 1981.

4 World Health Organisation. Abortion, a tabulation of available data on the frequency and monality of unsafe abortion. 2nd ed. Geneva: WHO, 1994.
CSM should rethink its approach for such announcements

EDrToR,-Klim McPherson estimates that in making its announcement when it did rather than waiting until all the relevant studies of third generation oral contraceptives had been published, the Committee on Safety of Medicines saved one life and prevented 80 cases of venous thromboembolism.' Our concern is not with the timing of the announcement in relation to the publication of peer reviewed papers or with the committee members' appraisal of the research evidence but with the manner in which the committee communicated the information to professionals and the public.

The announcement created much public anxiety and put great pressure on primary care services. Professionals working under pressure are less likely to be able to explain difficult concepts such as risk to their patients and more likely to make mistakes. Patients under stress are more likely to make the wrong decisions and less likely to be able to look after themselves. The public's health depends on individual people looking after themselves in a myriad of small ways every hour of every day. Anything that interferes with that process increases the risk of wrong decisions being made, resulting, for example, in an increase in unwanted pregnancies.

The fact that these risks are not easy to quantify does not mean that they are less important than the risk of thromboembolism or that the committee can ignore its responsibility for creating them. A phrase in McPherson's editorial perhaps holds the clue to at least one of the motivating forces in the committee's decision: "disproportionate fear of litigation." The committee members would be more likely to be blamed in court for a thromboembolic event than for complications of a termination of pregnancy that occurred because of a panic decision to stop taking oral contraceptives. Thus the committee's decision was the public health equivalent of the practice of defensive clinical medicine. It was more comfortable for the professionals but more distressing for the public.

This is not the first time that such problems have arisen. Perhaps in future a more proactive approach should be considered. The Committee on Safety of Medicines could ensure that its announcements are preceded by the development of written material for the public, to be distributed alongside the usual professional communications. The information would need to be prepared by professionals used to developing health education material and would need to be easily reproducible locally. If the committee made future announcements in this way it would support health professionals and encourage informed decision making by the public rather than create panic and confusion.

SARAH STEWART-BROWN Director CELIA PYPER

Health Services Research Unit, Associate member

Department of Public Health and Primary Care, University of Oxford,

Oxford $0 \mathrm{X}_{2} 6 \mathrm{HE}$

1 McPherson $\mathrm{K}$. Third generation oral contraception and venous thromboembolism. $B M F$ 1996;312:67-8. (13 January.)

\section{$12 \%$ Of women stopped taking their pill immediately they heard CSM's warning}

EDrroR,- - In his editorial on third generation oral contraceptives and the Committee on Safety of Medicines' actions Klim McPherson states, "We have no clear idea how many women responded to the committee's advice and in what way." I recently undertook a postal survey of all the women aged over 18 who had been prescribed a third generation pill in the past year in my practice (total population 9651). A total of 324 questionnaires were sent out, and $172(53 \%)$ women replied. Twenty women (12\%) said that they had stopped taking their pill on the day that the Committee on Safety of Medicines announced its warning, despite clear publicity that women should not do this. ${ }^{2}$ My finding must have implications for the way in which messages are handled by the committee in the future.

SALLY HOPE

The Surgery, General practitioner

Oxfordshire OX20 IUD

1 McPherson, K. Third generation oral contraception and venous thromboembolism. BMF 1996;312:68-9. (13 January.)

Committee on Safety of Medicines. Combined oral contraceptives and thromboembolism. London: CSM, 1995.

\section{How one clinic's practice conforms with CSM's advice}

EDITOR,-In seeking to interpret for women the recent research showing an approximate doubling of the relative risk of venous thromboembolic disease in women taking oral contraceptives containing gestodene or desogestrel ${ }^{1}$ we need ways in which to explain the complicated and subtle statistical evidence.

John Guillebaud contends that we can reassure a woman who has been taking combined ora contraceptives for a considerable time that "as she has not had a venous thrombosis in these years she is particularly unlikely to have one,"2 but I do not believe that this view is evidence based. The World Health Organisation's recent study concludes that "duration of current episodes of use and lifetime duration of use did not effect the risk estimates of VTE [venous thromboembolic disease]."

It is important to get the risks into perspective. The combined oral contraceptives are safe and effective for many women. The recently published data confirm the low risk of venous thromboembolic disease associated with combined pills containing norethisterone or norgestrel, at $15 / 100000$ women per year. The risk for pills containing desogestrel or gestodene is reported as around 30/100 000 women per year. ${ }^{3}$

In the service in which I work we now reserve pills containing desogestrel or gestodene for those few women who, having had unacceptable side effects while taking other combined formulations, do not wish to consider an alternative contraceptive method and have discussed and understand the current estimation of risks. Women who have been happy with pills containing gestodene or desogestrel but have never tried any others are being offered the opportunity to change to formulation that we now believe confers less risk. In our experience most women in this situation are pleased to be offered the opportunity of minimising their risks. We discuss with the women how to change between formulations without diminishing contraceptive cover, and we record careful notes of the clinical consultations. We do not prescribe pills containing desogestre or gestodene as first line choices for women with no experience of oral contraception, except occasionally to women with severe acne. We believe that this practice is in line with the current research evidence and the Committee on Safety of Medicines' advice.

In our attempts to interpret new research evidence to inform our clinical practice and to present clear information to those who seek our advice and treatment we must guard against stepping over the boundary into untested hypotheses or wishfu conjecture. 
1 World Health Organisation Collaborative Study of Cardiovascular Disease and Steroid Hormone Contraception. Venous thromboembolic disease and combined oral contraceptives: results of international multicentre case-control study. Lancet 1995;346:1575-82

2 Guillebaud J. Scare over oral contraceptives. BMf 1995;311: 1638-9. (16 December.)

3 Faculty of Family Planning and Reproductive Health Care and Family Planning Association. Response for doctors to the Committee on Safety of Medicines' letter of 18th October 1995. London: FFPRHC, 1995.

4 Committee on Safety of Medicines. Combined oral contraceptives and thromboembolism. London: CSM, 1995.

\section{Postmarketing surveillance does not catch all adverse events}

EDITOR,-H B M Reijnen and W J Atsma, of Organon Pharmaceuticals, use data from the company's product surveillance database to show that the risk of venous thromboembolism with the pills Marvelon and Mercilon, both of which contain desogestrel, is highest in the first few months of use and falls dramatically thereafter. ${ }^{1}$ The World Health Organisation's recent multicentre case-control study refutes this suggestion: the duration of current use of oral contraceptives did not alter the risk. ${ }^{2}$

What is worrying about the authors' letter is the fact that the product surveillance database contains data on only 434 adverse drug events, fewer than 100 of which are venous thromboemboli. With over 36 million woman years of exposure to these two pills, we can conservatively estimate that the total number of venous thromboemboli is over 5000. Thus the manufacturer's database has information on less than $2 \%$ of all such cases. The impressive graph accompanying the letter is simply a reflection of poor reporting getting progressively worse.

The database is highly selective, and its use in this debate is misleading. It also calls into question the methods of postmarketing surveillance.

This whole episode should make us reflect on the quality of evidence that we expect in our practice and how we came to adopt the newer progestogens. Was it reasonable to base a wholesale change in our prescribing practices on surrogate markers of arterial disease? Had we been a little more critical we might also now have reliable information on the benefits or otherwise of these pills with regard to such important side effects as weight gain, breakthrough bleeding, acne, and headache. Ethics committees may now refuse randomised controlled trials of these pills.

PAUL O'BRIEN

Raymede Clinic,

London W10 5SH

1 Reijnen HBM, Atsma WJ. Scare over oral contraceptives. BMF 1995;311:1639. (16 December.)

2 World Health Organisation Collaborative Study of Cardiovascular Disease and Steroid Hormone Contraception. Venous thromboembolic disease and combined oral contraceptives: results of international multicentre case-control study. Lancet 1995;346:1575-82.

\section{Study's results do not apply to norgestimate}

EDrToR,-The issue of the $B M f$ published on 13 January contains two papers, an editorial, and two other items in which "third generation" oral contraceptives are discussed. At times third generation oral contraceptives are referred to as those containing gestodene, desogestrel, or norgestimate; at other times they are referred to as those containing gestodene or desogestrel. This may confuse readers.

In their paper on the risk of venous thromboembolic disorders associated with third generation oral contraceptives Walter $O$ Spitzer and colleagues clearly state that for the purpose of their analysis "third generation oral contraceptives were defined as products containing low doses of ethinylo- estradiol (usually $30 \mu \mathrm{g}$ or $20 \mu \mathrm{g}$ ) and either gestodene or desogestrel." In the same paragraph the paper makes clear that "preparations containing norgestimate were included with the second generation products, to retain consistency with the World Health Organisation analysis." Inexplicably, however, in the "key messages" box in the paper one of the key messages refers to norgestimate as a third generation oral contraceptive and another states that third generation oral contraceptives are associated with an increased risk of thromboembolism.

Norgestimate was not associated with an in creased risk of thromboembolism in this paper, and I am certain that the authors did not wish to imply otherwise. To prevent confusion among both doctors and women, care should be taken to avoid use of the broad term "third generation" in discussions of the recent publications on oral contraceptives and the risk of venous thromboembolism; rather, the names of the specific progestogens affected should be given.

R J DONNELLY Medical directo

Janssen-Cilag,

PO Box 79,

High Wycombe HP14 4HJ

1 Spitzer WO, Lewis MA, Heinemann, LAJ, Thorogood M MacRae KD on behalf of Transnational Research Group on Oral Contraceptives and the Health of Young Women. Third generation oral contraceptives and risk of venou thromboembolic disorders: an international case-contro study. $B M \mathcal{F}$ 1996;312:83-8. (13 January.)

\section{Full description of controls is needed in study}

EDrToR,-It is not uncommon to select controls from two sources because of concerns about the suitability of any one source. Superficial analysis of the data presented by Michael A Lewis and colleagues shows that hospital controls who use oral contraceptives are roughly three times less likely than community controls to use third generation pills (table 1). ${ }^{\prime}$ Naively, one might conclude

Table 1-Use of oral contraceptives by hospita and community controls

\begin{tabular}{lccc}
\hline & $\begin{array}{c}\text { No } \\
\text { current } \\
\text { use }\end{array}$ & $\begin{array}{c}\text { Third } \\
\text { generation } \\
\text { pills }\end{array}$ & $\begin{array}{c}\text { Second } \\
\text { generation } \\
\text { pills }\end{array}$ \\
\hline Cases (hospital controls) & 173 & 11 & 26 \\
Community controls & 246 & 23 & 19 \\
\hline
\end{tabular}

$\chi^{2}$ Test for independence: $\chi^{2}=5.97,2 \mathrm{df}, \mathrm{P}=0.050$.

that third generation pills are a general tonic, particularly in comparison with second generation pills: women who use them are three times less likely to end up in hospital than those who do not use them (odds ratio 2.86 (exact $95 \%$ confidence interval 1.03 to $8 \cdot 1$ )).

Such a result should not, of course, be taken at face value but should lead us to question carefully the selection of controls in this study. Surprisingly, no details of why the hospital controls were admitted to hospital are given, either in the authors' two reports ${ }^{12}$ or in the supplementary material posted on the Internet. The authors adjust their analyses for several variables, including age, body mass index, and smoking, but this seems only to increase the relative difference in the type of oral contraceptive used between the two groups (ratio of adjusted odds ratios 3.64).

By contrast, the authors of the World Health Organisation's paper on deep vein thromboembolism discuss their reasons for enrolling two types of controls and comment on the differences between these two groups in their study. ${ }^{3}$ Essentially, they found that a much higher proportion of hospital controls than controls based in general practice used oral contraceptives but that the ratio of use of third to second generation pills was about the same in both groups. One might guess that hospital controls would better match for differences in use of hospital services whereas general practice controls would better match for variations in prescribing practice.

If controls are well chosen and the study is designed with adequate power there should be little difference in the distribution of the exposure of primary interest between the two groups. Particularly when this is not the case, a published report should contain a full description of each set of controls together with some discussion of the potential biases.

The problem of the appropriateness of a given set of controls serves to remind us of the pitfalls of observational studies and that they are a poor substitute for randomised controlled clinical trials.

PETER SASIENI Research scientist

Department of Mathematics, Statistics, and Epidemiology, Imperial Cancer Research Fund,

PO Box 123,

London WC2A 3PX

1 Lewis MA, Spitzer WO, Heinemann LAJ, MacRae KD, Bruppacher R, Thorogood $M$ on behalf of Transnationgl Research Group on Oral Contraceptives and the Health of Young Women. Third generation oral contraceptives and risk of myocardial infarction: an international case-control srudy. $B M \mathcal{F}$ 1996;312:88-90. (13 January.)

2 Spitzer WO, Lewis MA, Heinemann LAI, Thorogood M MacRae KD on behalf of Transnational Research Group on Oral Contraceptives and the Health of Young Women. Third generation oral contraceptives and risk of venous Third generation oral contraceptives and risk of venous thromboembolic disorders: an internat
study. $B M 7$ 1996;312:83-8. (13 January.)

study. BMF 1996;312:83-8. (13 January.)
World Health Organisation Collaborative Study of CardiovascuWorld Health Organisation Collaborative Study of Cardiovascu-
lar Disease and Steroid Hormone Contraception. Effect of lar Disease and Steroid Hormone Contraception. Effect of
different progestogens in low oestrogen oral contraceptives on venous thromboembolic disease. Lancet 1995;346:1582-8.

\section{Authors' reply}

EDrToR,-Peter Sasieni is correct in asserting that a randomised controlled clinical trial is stronger than an observational case-control study. When concerns exist about the safety of drugs that are already being marketed, however, randomised controlled trials are seldom feasible. Most issues of drug safety concern very small risks. In the case of venous thromboembolic disease, a rough estimate of the baseline population risk is one event in 10000 woman years. Given this annual incidence of 0.0001 in unexposed women, with a two tailed $\alpha$ of 0.05 , a $\beta$ of 0.2 , and a ratio of controls to exposed subjects of $1: 1$, each group would need to contain about 300000 women to detect a relative risk of 2 and about 60000 women to detect a relative risk of $4 .{ }^{1}$ These sample sizes clearly render a randomised controlled trial impractical. The background incidence of acute myocardial infarction among this population is even smaller than that of deep vein thrombosis, so a case-control approach is mandatory. Even a cohort study is almost impossible except with large computerised databases. ${ }^{2}$

A major difficulty with case-control studies is, as Sasieni states, the appropriate choice of controls. This is why we planned two sets of controls in the transnational study, to reflect the use of oral contraceptives in the general population and in hospital controls. Contrary to Sasieni's belief, the details of the controls and the rationale for choosing them were published before we started our fieldwork. ${ }^{3}$ The protocol stated our intention of collecting 200 cases of acute myocardial infarction, but the study was thwarted by publicity bias resulting from the actions of the Committee on Safety of Medicines. Largely because of public demand, we published the results at that point; they were based on the 153 cases of acute myocardial infarction that had accrued. Because we were aware of the discrepancy between the hospital 